\title{
Log-parabolic spectra and particle acceleration in the BL Lac object Mkn 421: Spectral analysis of the complete BeppoSAX wide band X-ray data set
}

\author{
E. Massaro ${ }^{1,2}$, M. Perri ${ }^{1,3}$, P. Giommi ${ }^{3}$, and R. Nesci ${ }^{1}$ \\ 1 Dipartimento di Fisica, Universitá La Sapienza, Piazzale A. Moro 2, 00185 Roma, Italy \\ 2 IASF - Sezione di Roma, CNR, via del Fosso del Cavaliere, 00113 Roma, Italy \\ 3 ASI Science Data Center, ESRIN, 00044 Frascati, Italy
}

Received 21 May 2003 / Accepted 18 September 2003

\begin{abstract}
We report the results of a new analysis of 13 wide band BeppoSAX observations of the BL Lac object Mkn 421. The data from LECS, MECS and PDS, covering an energy interval from 0.1 to over $100 \mathrm{keV}$, have been used to study the spectral variability of this source. We show that the energy distributions in different luminosity states can be fitted very well by a $\log$-parabolic law $F(E)=K\left(E / E_{1}\right)^{-\left(a+b \log \left(E / E_{1}\right)\right)}$, which provides good estimates of the energy and flux of the synchrotron peak in the SED. In the first four short observations of 1997 Mkn 421 was characterized by a very stable spectral shape, with average values $a=2.25$ and $b=0.45$, independently of the source brightness and of the fact that the source luminosity was increasing or decreasing. In the observations of 1998 smaller values for both parameters, $a \simeq 2.07$ and $b \simeq 0.34$, were found and the peak energy in the SED was in the range $0.5-0.8 \mathrm{keV}$. The observations of May 1999 and April-May 2000 covered runs of a duration of several days and provided a very high number of events for all the instruments. The resulting spectral fits were then limited by some instrumental systematics. Also in these cases, the log-parabolic model gave a satisfactory description of the overall SED of Mkn 421. In particular, in the observations of spring 2000 the source was brighter than the other observations and showed a large change of the spectral curvature. Spectral parameters estimates gave $a \simeq 1.8$ and $b \simeq 0.19$ and the energy of the maximum in the SED moved to the range $1-5.5 \mathrm{keV}$.

We give a possible interpretation of the log-parabolic spectral model in terms of particle acceleration mechanisms. An energy distribution of emitting particles with curvature close to the one observed can be explained by a simple model for statistical acceleration with the assumption that the probability for a particle to increase its energy is a decreasing function of the energy itself. A consequence of this mechanism is the existence of a linear relation between the spectral parameters $a$ and $b$, well confirmed by the estimated values of these two parameters for Mkn 421.
\end{abstract}

Key words. radiation mechanisms: non-thermal - galaxies: active - galaxies: BL Lacertae objects: individual: Mkn 421 $\mathrm{X}$ rays: galaxies

\section{Introduction}

BL Lac objects (and Blazars in general) are Active Galactic Nuclei (AGN) having a polarised and highly variable nonthermal continuum emission extending from radio to $\gamma$-rays. Their typical Spectral Energy Distribution (SED) is generally described by a double bump structure generated by two emission components. Current models consider the low frequency bump produced by synchrotron radiation (SR) from relativistic electrons in a jet closely aligned to the line of sight, and the high frequency bump produced by inverse Compton (IC) scattering. The peak frequency of the first bump typically ranges from the Infrared to the Optical for the so called Low-energy peaked BL Lac (LBL) objects while it is located

Send offprint requests to: E. Massaro,

e-mail: enrico.massaro@uniroma1.it in the UV-X ray bands for the High-energy peaked BL Lac (HBL) (Padovani \& Giommi 1995). To model the shape of these bumps in the $\log \left(v F_{v}\right)$ vs. $\log v$ plots, over frequency intervals extending through several decades, different analytical laws have been used. These laws are generally based on combinations of power laws, in some cases with an exponential cutoff, and contain several parameters.

Mkn 421 is a nearby $(z=0.031)$ BL Lac object classified as an HBL source because the energy of the synchrotron peak in the SED is higher than $0.1 \mathrm{keV}$. The spectrum presents a very well marked curvature and the flux changes are generally characterized by a high spectral variability (Fossati et al. 2000a). The high energy emission of Mkn 421 extends to the $\mathrm{TeV}$ range, where it was the first AGN to be discovered by the Whipple Observatory (Punch et al. 1992). Mkn 421 has been one of the main targets of multifrequency observational 
campaigns from ground and space observatories. In particular, BeppoSAX observed this source many times, but, because of the large amount of data collected in these occasions and the complexity of their analysis, a complete report of all these observations has not been published yet.

We present here a new study of the spectral properties of the Mkn 421 SEDs based on the entire collection of the BeppoSAX observations performed with the Narrow Field Instruments (NFIs) on board this satellite: LECS (0.1-10 keV) (Parmar et al. 1997), MECS (1.3-10 keV) (Boella et al. 1997) and PDS (13-300 keV) (Frontera et al. 1997). Some of these observations have already been analysed by other authors while a spectral analysis of 1999 and 2000 observations is presented here for the first time.

A time and spectral analysis of the 1997 and April 1998 BeppoSAX observations of Mkn 421 was presented by Fossati et al. (2000a, 2000b), while some results of the observation performed in June 1998 were presented by Malizia et al. (2000). To fit the spectra these authors used a modified formula of a continuous combination of two power laws, including several parameters whose physical interpretation is not direct. Furthermore Fossati et al. (2000b) used in their spectral fits only the LECS and MECS data and considered the PDS data separately, while fitting all these data at the same time could be more useful for a wide band description of the spectral shape.

In the first part of this paper (Sects. 3 to 5) we show that a simple log-parabolic law generally gives a very good description of the X-ray peak in SED of Mkn 421 also when it is applied to the data of the three NFIs (LECS, MECS and PDS) considered together. The fact that the synchrotron emission of BL Lac objects is generally well described by a log-parabolic law was found by Landau et al. (1986) in the analysis of several wide band observations (from the millimetric band to UV) of a sample of LBL sources, but these authors did not provide a physical explanation, based on the widely accepted emission model, for this type of spectral distribution. More recently, this spectral model was also used by Krennrich et al. (1999) to describe the spectral curvature of $\mathrm{Mkn} 421$ in the $\mathrm{TeV}$ range. In the second part (Sect. 6) of this paper we show that a logparabolic law is naturally expected in the framework of statistical acceleration when a more general and simple assumption about the particle probability to increase its energy is introduced. We derive also, from a simple phenomenological approach, the relations between the spectral parameters and the general properties of the acceleration mechanism. A further advantage of the log-parabolic law is that it can be used to estimate several useful quantities, like the peak frequency and bolometric flux of the spectral component, in a much simpler way than other models.

During the BeppoSAX pointing of April 1998, we also performed nearly simultaneous optical photometric observations. We use these data to study how the emission in this frequency range can be linked to the X-ray spectral distribution and show that simple extrapolations do not provide acceptable representations of the SED, while a log-parabolic model can help to disentangle the possible contributions from different emission components.
Finally we recall that the log-parabolic law has been already used by us to represent the broad band SED, from the optical to X rays, of some other BL Lac objects like OJ 287, MS 1458+22 (Massaro et al. 2003), 3C 66A and ON 325 (Perri et al. 2003). We also successfully applied this model in the analysis of the BeppoSAX X-ray data of Mkn 501 and the results will be presented in a subsequent paper.

\section{Observations and data reduction}

BeppoSAX observed Mkn 421 on 13 occasions: 7 times in April and May 1997, 3 times in April and June 1998, 1 time in May 1999 and 2 times in April and May 2000. In all the observations performed in 1997 the MECS operated with three detectors, while in those performed in the subsequent years only two detectors were operating. The logs of all these observations, the net exposure times for the three NFIs considered in our analysis and the PDS count rates in the $15-90 \mathrm{keV}$ energy band are given in Table 1. Observations were generally concentrated in time windows of several days: the first one had a total duration of ten days from the end of April to the beginning of May 1997. The pointings of 1999 and 2000 were much longer than those of the previous periods. In May 1999 Mkn 421 was observed for about 4 days and a longer uninterrupted run was from April 26 to May 3, 2000, followed by another observation started on May 9 and lasted more than 3 days. In all the observations the source showed a high variability, characterized by an irregular oscillating behaviour. The time analysis of the 1997 and April 1998 data has been presented by Fossati et al. (2000a) and Zhang (2002). Malizia et al. (2000) reported the light curves of June 1998 and those of the long observations of April and May 2000 are shown in a paper by Tanihata et al. (2003). We do not report in the present paper the 1997 and 1998 light curves and recall only that Mkn 421 was always observed to vary with a count rate amplitude of a factor of about 2 . In some short pointings the flux showed a regular trend: for instance on 1997 April 29 and May 3 the count rate decreased for the entire duration of the observation, while on 1997 May 1 it was increasing.

Standard procedures and selection criteria were applied to the data to avoid the South Atlantic Geomagnetic Anomaly, solar, bright Earth and particle contamination using the SAXDAS (v. 2.0.0) package. Data analysis was performed using the software available in the XANADU Package (XIMAGE, XRONOS, XSPEC). The images in the LECS and MECS showed a bright pointlike source. Events for spectral and timing analysis were extracted following the standard procedure currently in use at the BeppoSAX Science Data Center (SDC): data were selected in circular regions, centred at the source position, with a radius of $8^{\prime}$ for the 1999 and 2000 observations, in which the count rate was very high, and $6^{\prime}$ and $4^{\prime}$ for the LECS and MECS, respectively, in the other observations. The response matrices and arf files used in our analysis have been taken from the BeppoSAX SDC ftp server (September 1997 release) and background spectra were taken from the blank field archive. 
Table 1. BeppoSAX observation log of Mkn 421.

\begin{tabular}{|c|c|c|c|c|c|c|}
\hline Date & Start UT & End UT & LECS exp. (s) & MECS exp. (s) & PDS exp. (s) & PDS count rate ${ }^{1}\left(\mathrm{~s}^{-1}\right)$ \\
\hline $1997 / 04 / 29$ & 04:02 & $14: 42$ & 11562 & 23283 & 18388 & $0.21 \pm 0.04$ \\
\hline $1997 / 04 / 30$ & 03:19 & $14: 42$ & 11379 & 23975 & 22079 & $0.16 \pm 0.06$ \\
\hline 1997/05/01 & 03:17 & $14: 42$ & 11171 & 23713 & 21748 & $0.13 \pm 0.06$ \\
\hline $1997 / 05 / 02$ & $04: 10$ & 09:41 & 4417 & 11368 & 10594 & $0.31 \pm 0.08$ \\
\hline $1997 / 05 / 03$ & $03: 24$ & 09:41 & 4326 & 11672 & 10734 & $0.10 \pm 0.08$ \\
\hline $1997 / 05 / 04$ & $03: 25$ & $09: 45$ & 4880 & 12177 & 11168 & $0.16 \pm 0.08$ \\
\hline $1997 / 05 / 05$ & 03:32 & $09: 45$ & 4971 & 11911 & 10946 & $<0.08$ \\
\hline $1998 / 04 / 21-22$ & $01: 52$ & $03: 13$ & 23620 & 29547 & 26676 & $0.51 \pm 0.05$ \\
\hline $1998 / 04 / 23-24$ & $00: 27$ & $06: 37$ & 27188 & 34656 & 30923 & $0.41 \pm 0.05$ \\
\hline $1998 / 06 / 22-23$ & $07: 16$ & $02: 21$ & 11279 & 32516 & 27824 & $0.45 \pm 0.05$ \\
\hline 1999/05/04-08 & $18: 16$ & 03:21 & 62868 & 121813 & 114693 & $0.14 \pm 0.02$ \\
\hline $2000 / 04 / 26-/ 05 / 03$ & $17: 36$ & $02: 13$ & 140374 & $127702^{2}$ & 151251 & $3.34 \pm 0.03$ \\
\hline 2000/05/09-12 & 04:09 & 07:06 & 70070 & 139376 & 131768 & $2.55 \pm 0.02$ \\
\hline
\end{tabular}

1 15-90 keV energy band.

${ }^{2}$ MECS not operating in the second half of the observation.

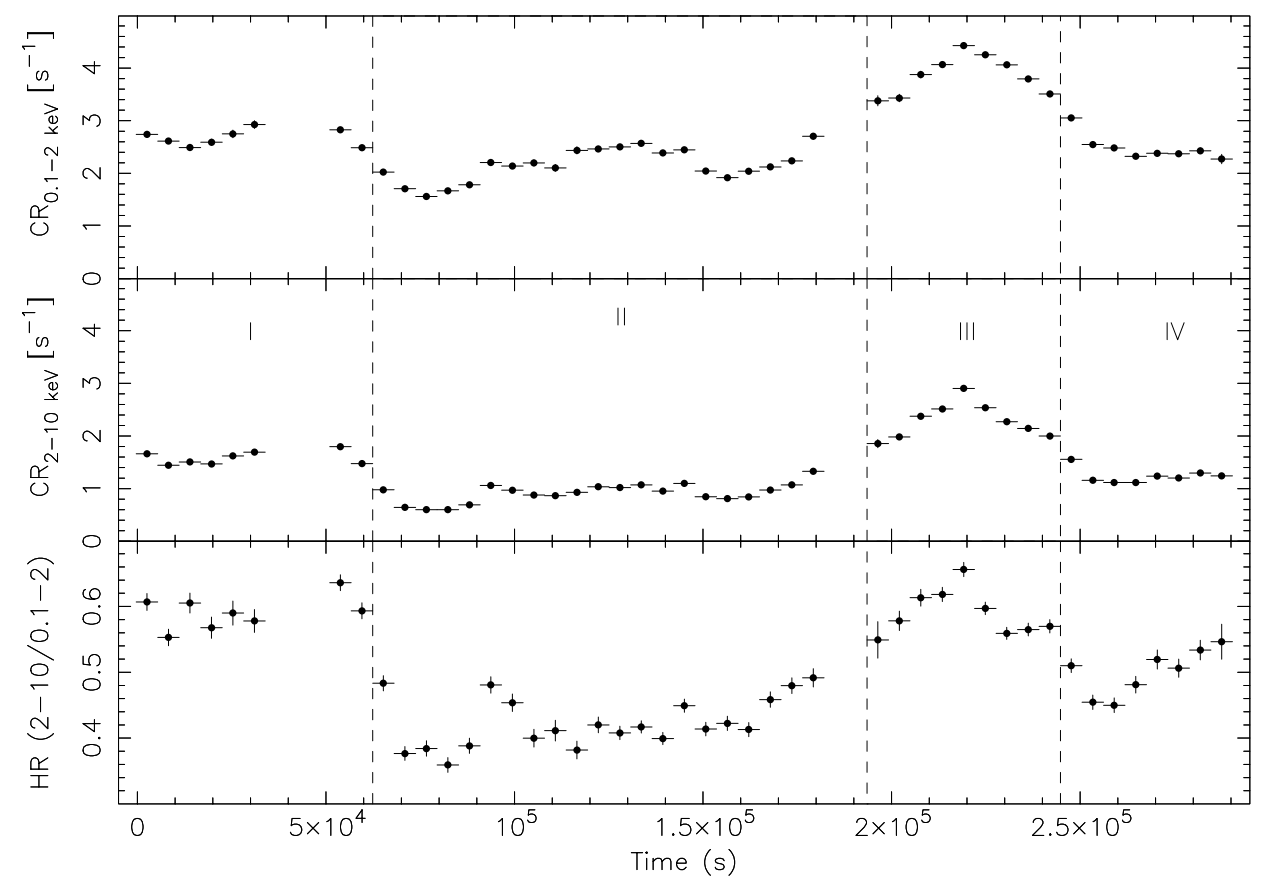

Fig. 1. The LECS (upper panel) and MECS (central panel) light curves of Mkn 421 during the observation of May 4-8, 1999. Data are binned in intervals of $5700 \mathrm{~s}$, approximately corresponding to a BeppoSAX orbit. The vertical scale is the same in the two panels to show the different amplitudes of the intensity variations. In the lower panel the corresponding hardness ratio (HR) is plotted. The dashed vertical lines indicate the four time segments considered in the spectral analysis.

The light curves in the LECS $(0.1-2.0 \mathrm{keV})$ and MECS (2.0-10 keV) bands of the observations of May 1999, April and May 2000 and the corresponding hardness ratios (HR) are shown in Figs. 1, 2 and 3, respectively. The time binning used in these plots corresponds to one BeppoSAX orbit (about 5700 s) and therefore it is not possible to see the hard lags shorter than 3 ks reported by Fossati et al. (2000a) and Zhang (2002). The count rate changes appear simultaneous in the two instruments, but the amplitudes are clearly different: the vertical scales of the LECS and MECS light curves are the same to make clearer these differences. Note that the same modulation appears in the HR light curves, confirming this amplitude difference.

In Fig. 1a strong flare is present in the second half of the observation. The rising portion lasted about $6.4 \times 10^{4} \mathrm{~s}$ and in this time the MECS count rate increased by a factor of 3.5 , while 


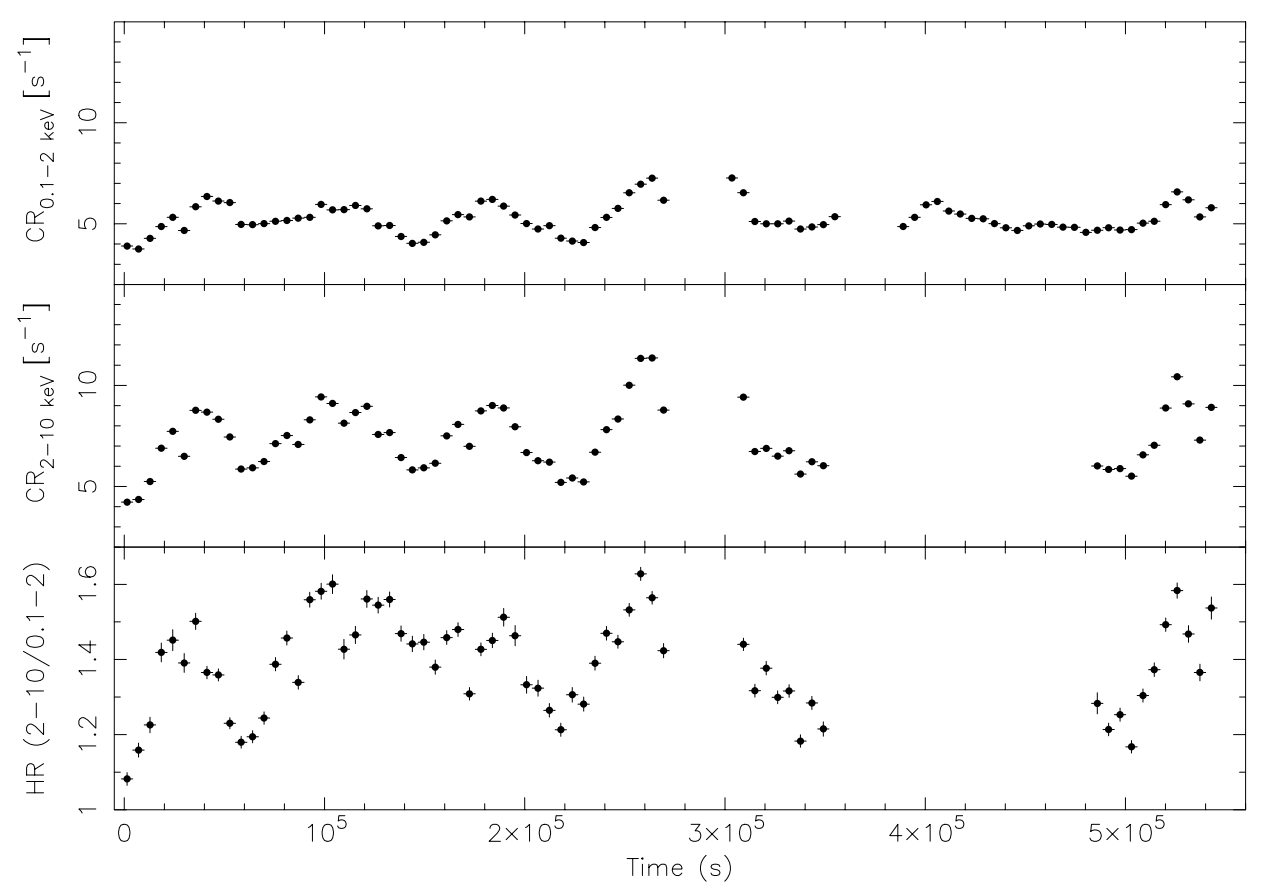

Fig. 2. The LECS (upper panel) and MECS (central panel) light curves of Mkn 421 during the observation of April 26-May 3, 2000. In the lower panel the corresponding hardness ratio (HR) is plotted. MECS was not operating in the time interval from 360000 to $480000 \mathrm{~s}$ after the beginning of the observation. Only the data taken in the first $360000 \mathrm{~s}$ (April 26-30) were used in the spectral analysis. Data are binned in intervals of $5700 \mathrm{~s}$, approximately corresponding to a BeppoSAX orbit. The vertical scale of the two panels are equal to show better the different intensity variations.

that of the LECS increased by the smaller factor of 2.3 ; the decay time was only about $20 \%$ shorter, indicating that there is no large difference in the rising and dimming phases of the flare.

The different amplitude ratios in the LECS and MECS intensities imply that this type of flux variations are generally associated with spectral changes, as also shown by the HR variations with time, and this makes more complex the spectral analysis. Similar behaviours are apparent also in the light curves of the two long observations performed in April-May and May 2000, shown in Figs. 2 and 3. In the course of the former observation Mkn 421 showed an oscillating behaviour with a typical recurrence time of $\sim 1$ day. Again, the same pattern is present in the light curve of May 2000 (Fig. 3), but in the last part of the observation two stronger flares are present with a very fast decay, a factor of 2.9 in $\sim 10^{4} \mathrm{~s}$ in the MECS band.

A relevant point that we want to stress when working with spectra obtained with two or three NFIs of BeppoSAX is that of the right choice of the inter-calibration factors between MECS with LECS $\left(f_{\mathrm{LM}}\right)$ and PDS $\left(f_{\mathrm{MP}}\right)$. The accurate ground and the in-flight calibrations were used to establish the admissible ranges for these two factors which are: $0.7 \leq f_{\mathrm{LM}} \leq 1.0$ and $0.77 \leq f_{\mathrm{MP}} \leq 0.93$, the latter reduced to $0.86 \pm 0.03$ for sources with a PDS count rate higher than $2 \mathrm{ct} / \mathrm{s}$ (Fiore et al. 1999). The use of intercalibration factors outside these ranges can affect the evaluation of the actual wide band spectral distribution, so it is not correct to consider them as completely free parameters in the fitting procedure and to fix their values for adjusting some results. In particular, when a spectrum has a well defined intrinsic curvature, like in Mkn 421, a not proper choice of the intercalibration factors introduces a bias in the spectral estimate. In our analysis we checked accurately that the best values of these two parameters were in the nominal ranges.

An important problem that we faced in evaluating the best fits originates from the accuracy of the response matrices and instrumental parameters. In the case of the LECS a large feature is present in the energy range around the carbon edge $(0.29 \mathrm{keV})$, and for long duration observations with a very high integrated source signal we found statistically significant residuals with respect to any smooth model (see Sect. 5 for the case of 1999 observation), already noticed by Fossati et al. (2000b) and Malizia et al. (2000). This feature does not change also using the 2000 release of the LECS response matrices. We verified that the same residuals are also detectable in the spectra of other sources with a high statistics like Mkn 501 and 3C 273. The most likely interpretation is that they are due to instrumental effects like either a not very precise evaluation of the LECS effective area in the region of the carbon edge or a moderate gain shift. In the case of high statistics spectra we have two undesired effects: $i$ ) the $\chi^{2}$ remains high even when the adopted model of the continuum gives a well acceptable representation of the general spectral behaviour; ii) the introduction of a bias in the best fit estimate of the spectral parameters. We verified that after introducing in the XSPEC best fit procedure a moderate gain correction for the LECS only, a gain increase of about $3 \%$ minus a bias of only $5 \mathrm{eV}$, the residuals are very largely reduced and much better $\chi^{2}$ values are obtained (see Sect. 5).

For a correct modelling of the spectral curvature it is very important to known the low energy absorption due to the interstellar gas. In our analysis we fixed this value to the galactic 


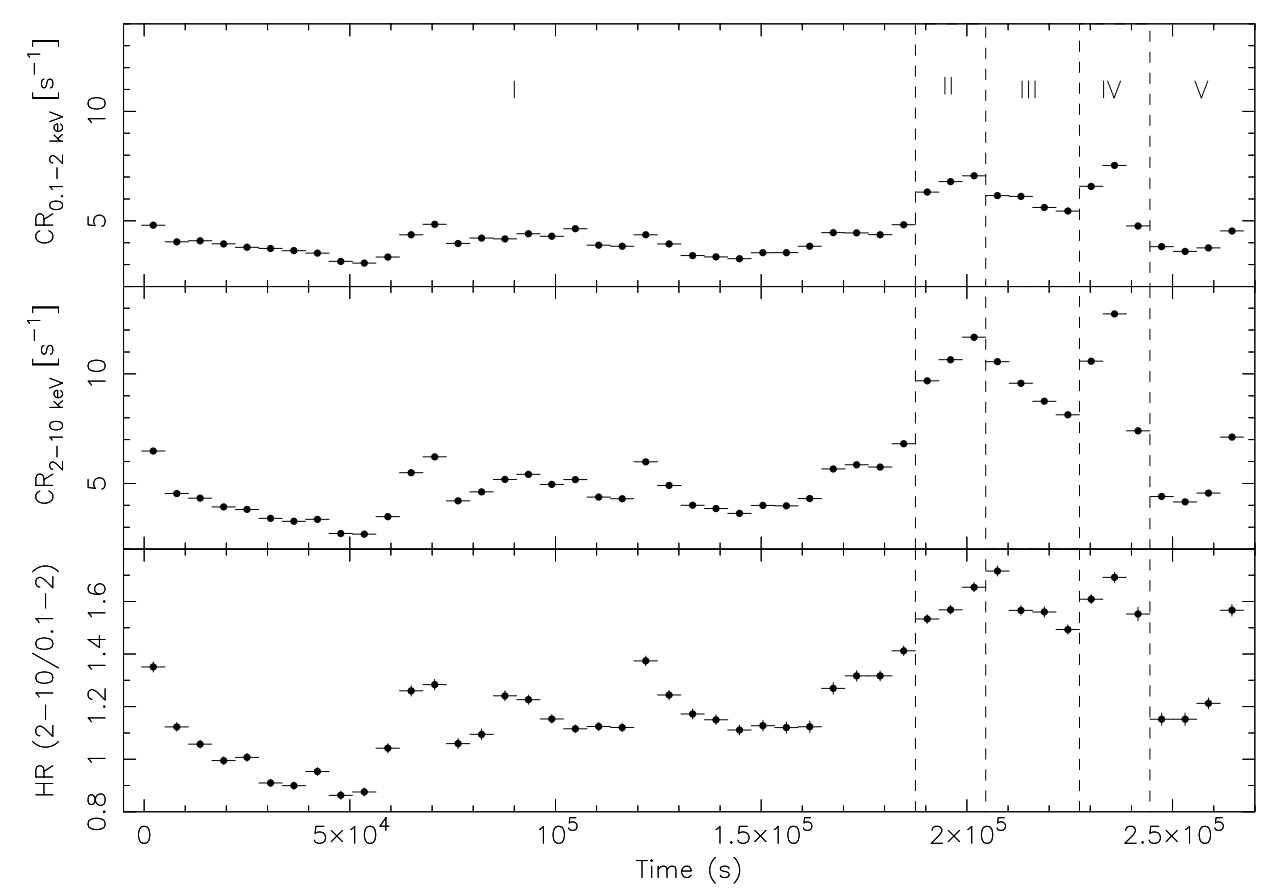

Fig. 3. The LECS (upper panel) and MECS (central panel) light curves of Mkn 421 during the observation of May 9-12, 2000. Data are binned in intervals of $5700 \mathrm{~s}$, approximately corresponding to a BeppoSAX orbit. The vertical scale is the same in the two panels to show the different amplitudes of the intensity variations. In the lower panel the corresponding hardness ratio (HR) is plotted. The dashed vertical lines indicate the five time segments considered in the spectral analysis.

column density equal to $N_{\mathrm{H}}=1.61 \times 10^{20} \mathrm{~cm}^{-2}$ as derived from the $21 \mathrm{~cm}$ measure by Lockman \& Savage (1995). One cannot exclude that a further absorption can be due to the host galaxy. However, high resolution optical images (Urry et al. 2000) do not give any firm indication for a large absorbing gas in the galaxian brightness profile and therefore we are confident that the above $N_{\mathrm{H}}$ is well representative of the actual value.

As already shown by Fossati et al. (2000b), the X-ray spectral distributions of Mkn 421 are remarkably curved. Best fits with simple power laws give largely unacceptable $\chi^{2}$. One can consider several different analytical models for describing these spectra, like a broken power law or a power law with an exponential cut-off (PL+EC model) at the energy $E_{\mathrm{c}}$ :

$$
F(E)=K E^{-\Gamma} \exp \left(-E / E_{\mathrm{c}}\right) \mathrm{ph} /\left(\mathrm{cm}^{2} \mathrm{~s} \mathrm{keV}\right) .
$$

This model is generally expected when the spectrum of the emitting electrons has a sharp or an abrupt upper cut-off in energy because of some limiting processes in the acceleration mechanisms. It has been considered by several authors and for this reason we decided to fit it to the data of Mkn 421. As it is shown in Sects. 4 and 5, the resulting $\chi^{2}$ are generally too large to be acceptable, indicating that the actual spectral curvature is milder than an exponential. Fossati et al. (2000b) considered a more complex spectral model given by a continuous combination of two power laws with photon indices $\Gamma_{-\infty}$ and $\Gamma_{+\infty}$, representative of the asymptotic spectral behaviours:

$F(E)=K E^{-\Gamma_{-\infty}}\left[1+\left(\frac{E}{E_{B}}\right)^{f}\right]^{\left(\Gamma_{-\infty}-\Gamma_{+\infty}\right) / f}$,

without introducing a restriction for their values. In principle it is necessary to evaluate the best values of five free parameters. Such a law actually provides acceptable fits, but it is not simple to relate the parameters' value to the physical quantities of the source model. Fossati et al. (2000b) did not evaluate the asymptotic spectral indices but derived the local slopes at four energies inside the BeppoSAX band to describe better the spectral curvature. They kept the value of $f$ fixed to 1 in the analysis of the 1997 data and to 2 in that of 1998, without a clear justification based on a physical model of the source.

As stated in the Introduction, in our analysis we preferred to describe the curved spectrum of the photon flux with the following log-parabolic (Log- $P$ ) model:

$$
F(E)=K\left(E / E_{1}\right)^{-\left(a+b \log \left(E / E_{1}\right)\right)} \mathrm{ph} /\left(\mathrm{cm}^{2} \mathrm{~s} \mathrm{keV}\right) .
$$

We fixed the reference energy $E_{1}$ to $1 \mathrm{keV}$ and therefore the spectrum is completely determined only by the three parameters $K, a$ and $b$. This model has been already used with success to fit the spectrum of the Crab pulsar (Massaro et al. 2000) over several decades in energy and was also applied in the catalog of 157 X-ray SED of Blazars observed with BeppoSAX (Giommi et al. 2002). The main properties of the log-parabolic are briefly given in the following section.

\section{Main properties of the log-parabolic spectral distribution}

The log-parabolic model of Eq. (3) is one of the simplest way to represent curved spectra when they do not show a sharp high energy cut-off like that of an exponential. It has only one additional parameter with respect to a simple power law and it is also very useful to estimate other interesting quantities relevant for the physical modelling of the emission region. It is possible 
to define an energy dependent photon index $\Gamma(E)$, given by the log-derivative of Eq. (3):

$\Gamma(E)=a+2 b \log \left(E / E_{1}\right)$.

The parameter $a$ is the photon index at the energy $E_{1}$, while $b$ measures the curvature of the parabola: it is easy to demonstrate that the curvature radius at the parabola vertex is equal to $1 /|2 b|$. A good estimate of $b$ can be obtained only using wide enough energy ranges, particularly when its value is small.

Another useful form to describe the SED is the following:

$\log (v F(v))=\log \left(v_{\mathrm{p}} F\left(v_{\mathrm{p}}\right)\right)-b\left[\log \left(\frac{v}{v_{\mathrm{p}}}\right)\right]^{2}$,

where $v_{\mathrm{p}}=E_{\mathrm{p}} / h$ is the peak frequency corresponding to the maximum $v_{\mathrm{p}} F\left(v_{\mathrm{p}}\right)$ of the SED. These quantities can be easily computed from the spectral parameters $a, b$ and $K$ :

$E_{\mathrm{p}}=E_{1} 10^{(2-a) / 2 b}$

and

$$
\begin{aligned}
v_{\mathrm{p}} F\left(v_{\mathrm{p}}\right) & =\left(1.60 \times 10^{-9}\right) K E_{1} E_{\mathrm{p}}\left(E_{\mathrm{p}} / E_{1}\right)^{-a / 2}, \\
& =\left(1.60 \times 10^{-9}\right) K E_{1}^{2} 10^{(2-a)^{2} / 4 b} \mathrm{erg} /\left(\mathrm{cm}^{2} \mathrm{~s}\right)
\end{aligned}
$$

where the numerical constant is simply the energy conversion factor from $\mathrm{keV}$ to erg.

This distribution can be analytically integrated over the entire frequency range to estimate the bolometric flux. It is not difficult to show that the integral of Eq. (5) between the frequency limits 0 and $\infty$ can be transformed in that of a Gaussian function between $-\infty$ and $+\infty$. The final result is:

$F_{\text {bol }}=\sqrt{\pi \ln 10} \frac{v_{\mathrm{p}} F\left(v_{\mathrm{p}}\right)}{\sqrt{b}}=2.70 \frac{v_{\mathrm{p}} F\left(v_{\mathrm{p}}\right)}{\sqrt{b}}$.

Considering that typical values of $b$ range around $0.2-0.4$ (see the next section) one can obtain a rough estimate of the bolometric flux as $F_{\text {bol }} \simeq 5 v_{\mathrm{p}} F\left(v_{\mathrm{p}}\right)$.

Another advantage of the log-parabolic model is that the spectral curvature is characterised only by the parameter $b$, while in other models, like the continuous combination of two power laws of Eq. (2), it is function of several parameters. A limit of the model, however, is that it can represent only energy distributions symmetrically decreasing with respect to the peak frequency. More general models, to take into account a possible asymmetry, must include at least another parameter. For instance, if at low energies the spectrum follows a single power law, while the log-parabolic bending becomes apparent at energies higher than a critical value $E_{\mathrm{c}}$, one could use the following mixed model:

$F(E)=K\left(E / E_{1}\right)^{-\left(a+b \log \left(E_{c} / E_{1}\right)\right)}, E \leq E_{\mathrm{c}}$
$F(E)=K\left(E / E_{1}\right)^{-\left(a+b \log \left(E / E_{1}\right)\right)}, E>E_{\mathrm{c}}$

however it was not used in the present work since the peak energy of Mkn 421 is generally below $1 \mathrm{keV}$ and only limited data are available to detect a significant deviation from a symmetric distribution.
Table 2. Reduced $\chi^{2}$ values for the best fit spectra of Mkn 421 with a power law with an exponential cutoff and a log-parabola for the observations in 1997 and 1998.

\begin{tabular}{lccc}
\hline \hline Date & PL+EC & $\log P$ & d.o.f. \\
\hline $1997-04-29^{(1)}$ & 2.39 & 1.31 & 132 \\
$1997-04-30^{(1)}$ & 1.82 & 0.75 & 136 \\
$1997-05-01^{(1)}$ & 2.42 & 1.06 & 132 \\
$1997-05-02^{(1)}$ & 1.41 & 0.86 & 127 \\
$1997-05-03^{(1)}$ & 1.32 & 0.97 & 100 \\
$1997-05-04^{(1)}$ & 1.55 & 1.16 & 100 \\
$1997-05-05^{(1)}$ & 1.75 & 1.11 & 100 \\
& & & \\
$1998-04-21$ & 3.73 & 1.19 & 171 \\
$1998-04-23$ & 2.83 & 1.02 & 169 \\
$1998-06-22$ & 2.48 & 1.26 & 150 \\
\hline
\end{tabular}

(1) PDS data not included.

\section{The 1997-1998 observations}

\subsection{The X-ray spectral analysis}

In the analysis of the seven observations of spring 1997 we used only the LECS and MECS data, while those from PDS were not used in the analysis. In fact, the faint state of the source and the spectral steepening at high energies imply a count rate above $15 \mathrm{keV}$ much below the sensitivity of the instrument which is limited by confusion due to background sources. In the three observations of 1998, when the source mean flux was higher than in the previous year, PDS events up to the energy of 40-60 keV were also included in the spectral analysis.

An inspection of the light curves showed that time variability within each pointing was limited to about $30 \%$ or less and the hardness ratio was consistent with being constant. For this reason spectral fits were evaluated using the events accumulated in the entire duration of each observation thus allowing us to use the highest statistics for a precise estimation of the spectral parameters.

As mentioned in Sect. 2, we fitted the photon spectra using the log-parabolic model and obtained statistically acceptable results, much better than those obtained with a single or a broken power law. In Table 2 we report the reduced $\chi^{2}$ values and the corresponding d.o.f. for the power law with exponential cutoff and for the log-parabolic model: it is evident that the former model is generally not acceptable, while the latter gives reduced $\chi^{2}$ quite closer to unity. In particular the power law with exponential cutoff failed to fit the data of the 1998 observations when, because of the higher brightness of the source, there is a significant detection in the PDS data at energies above $13 \mathrm{keV}$.

In Table 3 we report the best fit parameters values for the log-parabolic law, $a, b$ and $K$, together with three derived parameters, namely the peak energy, the maximum of the SED and the bolometric flux of the SR component. Throughout this paper errors are given at 1 standard deviation confidence level for one interesting parameter $\left(\Delta \chi^{2}=1\right)$. Four examples of these fits at different epochs, including those of the highest and lowest intensity are shown in Fig. 4. The best fits of the other 
Table 3. Best fit spectral parameters of the log-parabolic model for the 1997-1998 observations of Mkn 421.

\begin{tabular}{cccccccc}
\hline \hline Date & $a$ & $b$ & $K$ & $E_{\mathrm{p}}(\mathrm{keV})$ & $v_{\mathrm{p}} F\left(v_{\mathrm{p}}\right)^{(2)}$ & $F_{\mathrm{bol}}^{(2)}$ & $F_{2-10 \mathrm{keV}}^{(2)}$ \\
\hline $1997-04-29^{(1)}$ & $2.25(0.01)$ & $0.45(0.01)$ & $7.21(0.09) \times 10^{-2}$ & $0.53(.02)$ & $1.25(.02)$ & $5.0(.1)$ & $0.85(.02)$ \\
$1997-04-30^{(1)}$ & $2.26(0.01)$ & $0.47(0.01)$ & $7.25(0.09) \times 10^{-2}$ & $0.53(.01)$ & $1.26(.02)$ & $5.0(.1)$ & $0.83(.02)$ \\
$1997-05-01^{(1)}$ & $2.23(0.01)$ & $0.43(0.01)$ & $8.21(0.10) \times 10^{-2}$ & $0.54(.02)$ & $1.41(.02)$ & $5.8(.1)$ & $1.00(.02)$ \\
$1997-05-02^{(1)}$ & $2.25(0.01)$ & $0.43(0.02)$ & $1.02(0.02) \times 10^{-1}$ & $0.51(.02)$ & $1.78(.04)$ & $7.3(.2)$ & $1.23(.03)$ \\
$1997-05-03^{(1)}$ & $2.32(0.02)$ & $0.44(0.02)$ & $6.57(0.14) \times 10^{-2}$ & $0.43(.03)$ & $1.20(.03)$ & $4.9(.2)$ & $0.71(.03)$ \\
$1997-05-04^{(1)}$ & $2.50(0.02)$ & $0.48(0.02)$ & $4.96(0.13) \times 10^{-2}$ & $0.30(.02)$ & $1.07(.04)$ & $4.2(.2)$ & $0.41(.02)$ \\
$1997-05-05^{(1)}$ & $2.40(0.01)$ & $0.45(0.02)$ & $6.47(0.13) \times 10^{-2}$ & $0.36(.02)$ & $1.27(.03)$ & $5.1(.2)$ & $0.63(.02)$ \\
& & & & & & \\
$1998-04-21$ & $2.073(0.004)$ & $0.344(0.006)$ & $1.88(0.01) \times 10^{-1}$ & $0.78(.01)$ & $3.04(.02)$ & $13.9(.1)$ & $3.10(.02)$ \\
$1998-04-23$ & $2.219(0.004)$ & $0.373(0.007)$ & $1.35(0.01) \times 10^{-1}$ & $0.51(.01)$ & $2.33(.02)$ & $10.3(.1)$ & $1.78(.02)$ \\
$1998-06-22$ & $2.066(0.007)$ & $0.341(0.008)$ & $1.44(0.01) \times 10^{-1}$ & $0.80(.02)$ & $2.32(.02)$ & $10.7(.1)$ & $2.39(.02)$ \\
\hline
\end{tabular}

Errors are given at 1 sigma for one interesting parameter.

(1) PDS data not included.

(2) In units of $10^{-10} \mathrm{erg} \mathrm{cm}^{-2} \mathrm{~s}^{-1}$.

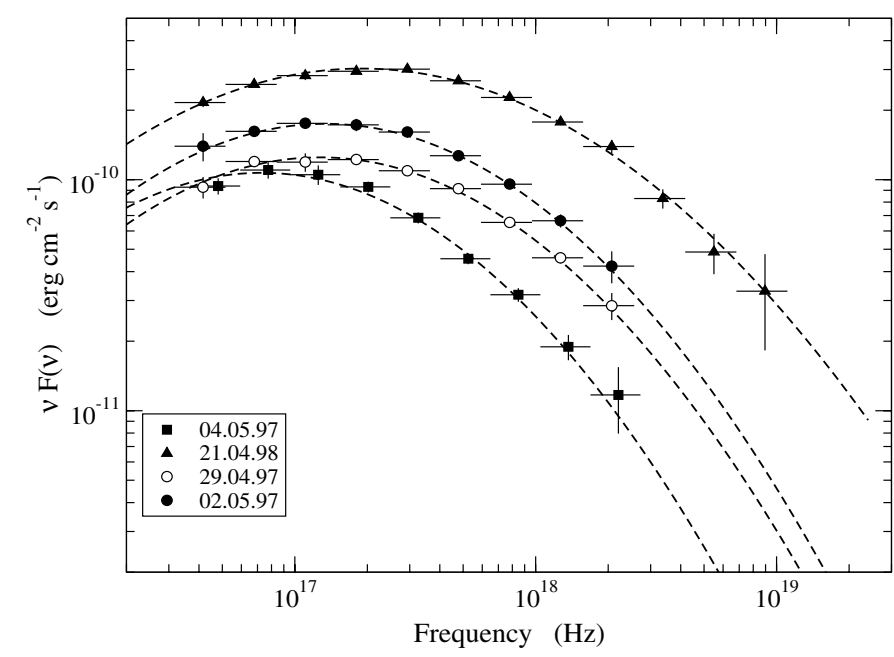

Fig. 4. The X-ray Spectral Energy Distributions of Mkn 421 in some of the BeppoSAX 1997-1998 observations deconvolved with a logparabolic law. Dashed lines are the best fit to the observed data.

observations are very similar and they are not shown in the figure for simplicity.

From the data of Table 3 we have important indications about the long term evolution of the spectral parameters. During the 7 day campaign of April-May 1997 the spectral curvature was remarkably high and very stable, with $b$ in the range $0.43-0.48$ with a mean value of 0.45 . Also $a$, the photon index at $1 \mathrm{keV}$, changed little: in particular, in the first four days it remained practically constant around the mean value of 2.25 and increased to $2.32-2.50$ in the last three days. In the same period the value of the normalisation $K$ instead changed more than a factor of 2 , indicating that these variations were not accompanied by large spectral changes. The SED was characterised by a rather low peak energy $E_{\mathrm{p}}$, which ranged in the narrow interval $0.30-0.54 \mathrm{keV}$ and by a maximum value $v_{\mathrm{p}} F\left(v_{\mathrm{p}}\right)$ between 1.07 and $1.78 \times 10^{-10} \mathrm{erg} \mathrm{cm}^{-2} \mathrm{~s}^{-1}$. The mean spectral shape remained practically unchanged independently of the fact that flux was decreasing (1997 April 29, May 3) or increasing (May 1 1997).

In $1998 \mathrm{Mkn} 421$ was brighter than in the preceding campaign: the highest count rate was measured on April 21 and resulted a factor of $\sim 4$ higher than in the lowest state of May 4, 1997. This increase of the total counts had the consequence that the systematic effects on the LECS spectra, described in Sect. 2, were more relevant than in the short observations of 1997 and the $\chi^{2}$ values were slightly higher, but always acceptable. Furthermore, we measured a signal in the PDS, up to $\sim 40-60 \mathrm{keV}$, with a better evaluation of the curvature parameter because of the more extended range. The SED maximum increased to values in the range $2.3-3.0 \times$ $10^{-10} \mathrm{erg} \mathrm{cm}^{-2} \mathrm{~s}^{-1}$, but this did not had a clear correspondence in the peak energy. It was $0.78 \mathrm{keV}$ in the first observation, when the flux from Mkn 421 was high to decrease to 0.51 after two days when the flux was about $30 \%$ lower. In the subsequent observation (June 1998), however, when the mean level of the source was practically the same of April 23, we found an $E_{\mathrm{p}}$ value of $0.80 \mathrm{keV}$ (see Table 3). Note also that the $b$ values decreased with respect to 1997, being in the range $0.34-0.37$. This smaller curvature is not due to an enhanced emission at higher energies in the PDS range, because it is also well measured using only the MECS data. According to Fossati et al. (2000b) on April 231998 Mkn 421 was found bright between 12 and $90 \mathrm{keV}$ with a quite hard spectrum: the photon index given by these authors $(2.35 \pm 0.25)$ is much smaller than that expected from the extrapolation of the logparabolic law with Eq. (4) and equal to 3.16, despite the large statistical uncertainty. We verified the count rates in the 12-40 and $40-90 \mathrm{keV}$ ranges and found $0.318 \pm 0.036$ and $0.102 \pm$ 0.034 respectively, in agreement within the statistical errors with those of Fossati et al. (2000b). Again a simple power law fit gives a flat spectrum. We note, however, that this count rate level, more than a factor of $\sim 2$ higher than that of April 21 when Mkn 421 was brighter, corresponds to a $v F(v)$ value of $\sim 3 \times$ $10^{-11} \mathrm{erg} \mathrm{cm}^{-2} \mathrm{~s}^{-1}$, moderately larger than the contribution from the cosmic background. We cannot exclude that this 
enhanced emission above $15 \mathrm{keV}$ might be due to some statistical fluctuation, although the interesting hypothesis that it could be an indication of a new spectral component emerging at higher energies cannot be excluded.

\subsection{Optical observations}

The low energy tail of the SR peak can extend in the UV-optical range. Observations at these frequencies are then useful to understand how wide is the range in which the log-parabolic model gives an acceptable description of the SED. However, a direct use of photometric data, in particular if they have been obtained with small aperture telescopes, is complicated by the fact that the nuclear emission from Mkn 421 is embedded in that of the bright host elliptical galaxy. To correct the measured data for the galaxian component it is necessary to know how much it contributes to the total flux within the used aperture. In literature data photometric apertures are not always indicated and it is not possible to apply the corrections for the host galaxy contribution to derive a reliable estimate of the flux from the nucleus.

We performed some photometric observations of Mkn 421 in the $B, V$ (Johnson) and $R$ (Cousins) bandpasses on April 24, 25 and 28 1998, just after the BeppoSAX observations, with the $0.70 \mathrm{~cm}$ reflector telescope of the University of Roma and IASF-CNR located at Monte Porzio and equipped with a backilluminated CCD camera (Site 501A). Standard stars were taken from Villata et al. (1998). In the three nights, the magnitudes of Mkn 421, within a photometric radius of 3.2 arcsec, remained substantially stable in a range smaller than 0.1 mag. The measured mean values are: $B=13.26 \pm 0.10, V=12.96 \pm$ $0.05, R=12.65 \pm 0.03$. The $V-R$ colour index is in a very good agreement with those measured in other occasions (see, for instance, Tosti et al. 1998).

We evaluated the host galaxy contribution using the brightness profile given by Urry et al. (2000), which gives for our photometric radius a magnitude $R_{\mathrm{gal}}=14.05$. The magnitudes in the other two bands were computed from the typical colours of an elliptical galaxy (Fukugita et al. 1995) and resulted equal to $B_{\text {gal }}=15.62$ and $V_{\text {gal }}=14.66$. The fluxes of the central component were then computed using the zero magnitude values from Mead et al. (1990) and obtained $F(R)=20.1 \pm 0.8$, $F(V)=18.8 \pm 1.3$ and $F(B)=18.7 \pm 1.9 \mathrm{mJy}$. The optical spectrum of the nuclear source is then quite flat with an energy index of $0.3 \pm 0.2$. This uncertainty is derived only from the photometric errors and it could be even greater if possible systematics, like those derived from the galaxy subtraction, are also properly taken into account.

In Fig. 5 we plotted the SED of Mkn 421 derived from these nearly simultaneous observations. It is clearly apparent that the optical and X-ray data cannot be described by a single component either with a power law or a log-parabolic distribution. The extrapolation of log-parabolic best fit of the X-ray data into the optical band gives fluxes much lower than the observed ones. Furthermore, assuming that the log-parabola could be extrapolated in the optical-UV range by a power law, as modelled by Eq. (9), our optical data cannot be fitted since the spectral

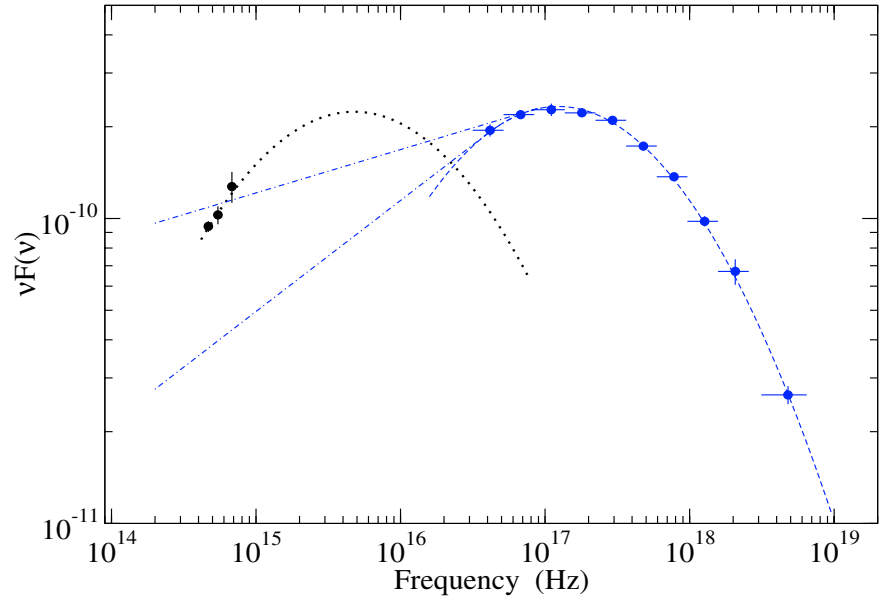

Fig. 5. The optical to X-ray Spectral Energy Distribution of Mkn 421 observed on 1998, April 23-24. Two log-parabolic spectral distributions for the optical and X-ray data sets are shown, together with possible power law extrapolations of X-ray data at optical frequencies. Notice that these extrapolations cannot match the photometric $R, V$, $B$ points.

slope is very different from the observed one. Conversely, also the power law extrapolation of the optical data to the X-ray range does not give the right fluxes and slope.

This result suggests that the optical emission does not belong to the same component of the $\mathrm{X}$ rays, although it could still be produced by the same electron population. In the same Fig. 5 we plotted a possible optical log-parabolic spectrum, with the same $b$ value of the X-ray data and the peak value adjusted to have a small contribution at energies higher than $\sim 1 \mathrm{keV}$ : the resulting peak frequency would then be around $7.5 \times$ $10^{15} \mathrm{~Hz}$ and the maximum in the SED comparable to or slightly higher than that observed in the $\mathrm{X}$ rays. An interesting consequence of this model is that the flux observed at energies lower than $\sim 0.5 \mathrm{keV}$ is due to the superposition of these two components, which can be variable with different amplitudes and time scales. This assumption can explain why the variation amplitudes measured from the LECS light curves are systematically smaller than those measured from the simultaneous MECS time series (see Figs. 1, 2 and 3). Finally, we stress that the presence of this low energy component can modify the spectral distribution producing a rather constant energy index close to unity.

\section{The 1999-2000 observations}

The three last campaigns in 1999 and 2000 provided long series of data with a very great number of events both in the LECS and MECS. Because of such high statistics, systematic effects of the detectors, usually negligible for other sources, become relevant and produce large $\chi^{2}$ values which can be improved by modifying the instrumental response very little. The comparison with a power law plus an exponential cut-off confirmed again that the log-parabolic law is better for the general modelling of the continuum. Spectral analysis was also applied to data subsets, selected either in particular time intervals or for 

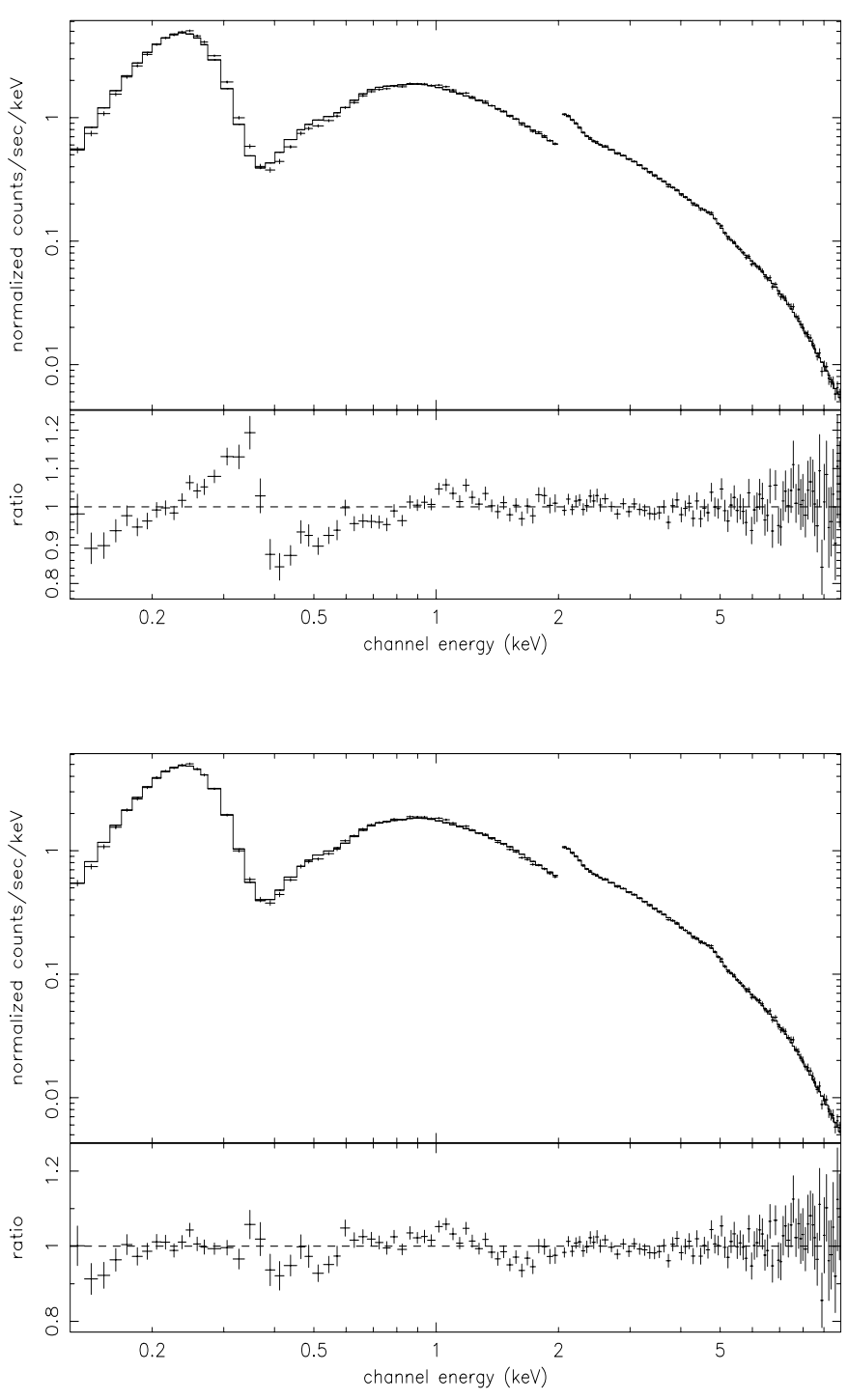

Fig. 6. Upper panel: best fit of the LECS and MECS data with a log-parabolic law of the 1999 BeppoSAX observation of Mkn 421 without gain correction. Note the large residuals of the LECS points between 0.2 and $0.6 \mathrm{keV}$ due to some systematics due to the near Carbon edge.

Lower panel: best fit of the same data after the gain adjustment of the LECS. The spurious features at low energies practically disappeared.

different average levels of count rate, to study the possible relations between $a$ and $b$ and other source parameters.

The best fit of the four day observation of May 1999 using the log-parabolic model applied to the LECS and MECS data gave the unacceptable reduced $\chi^{2}=3.12$ (139 d.o.f), as also evident from the data and residuals shown in Fig. 6. Similar results are also obtained for the 2000 observations. As we discussed in Sect. 2, this high $\chi^{2}$ is due to the large deviation between 0.3 and $0.4 \mathrm{keV}$, corresponding to the carbon edge feature in the detector effective area (Orr et al. 1997). Moreover, it is possible to see how this feature affects the LECS continuum over a quite large energy range. When the energy bins below $0.8 \mathrm{keV}$ are not included in the best fit the reduced $\chi^{2} \mathrm{de}-$ creases down to much better value of 1.25 (105 d.o.f.). As stated in Sect. 2, we tried to adjust the instrumental parameter to reduce the inconvenience due to this spurious features. We applied the XSPEC command GAIN to the LECS data correcting the energy channel relation with a linear law and found that the feature practically disappeared as shown in the lower panel of Fig. 6. To be sure that this correction does not alter the estimate of the spectral parameters, we evaluated their best fit values after eliminating the energy bins between 0.1 and $0.8 \mathrm{keV}$ and they generally remained unchanged within the statistical errors.

Large $\chi^{2}$ values are also obtained from the 1999-2000 MECS data. An inspection of the residuals shows that they are likely due to systematics in the response matrices of the instrument, which are much larger than statistical uncertainties because of the very high number of events. The accuracy of calibration of this instrument is indeed of about $2 \%$ (Fiore et al. 1999), and we verified that it is sufficient to take into account a systematic error of $1.5 \%$ to reduce the $\chi^{2}$ to well acceptable values.

We therefore decided to apply these corrections in the spectral analysis of all high statistics observations (1999-2000) and limited the useful LECS range to $0.1-2 \mathrm{keV}$.

\subsection{The 1999 observation}

During this observation the mean count rate level of Mkn 421 was intermediate between those measured in 1997 and 1998. The flux in the PDS band was therefore not high and these data were used in the spectral fit of the whole observation only up to $25 \mathrm{keV}$. Although the used range was narrower than in the 1997-1998, we detected a significant spectral curvature, with an average $b=0.42$, very similar to the results of 1997 . The value of $a$ is higher and determines a lower value of $E_{\mathrm{p}}$. The resulting SED, plotted in Fig. 7, is similar to those of the previous observations.

We evaluated the log-parabolic best fits also in four shorter time intervals to study the possible evolution of the SED. These intervals, identified by the roman numerals from I to IV, correspond to the following time window in the light curve of Fig. 1: I from 0 to $62000 \mathrm{~s}$, II from 62000 to $190000 \mathrm{~s}$, III from 190000 to $245000 \mathrm{~s}$ and IV from 245000 to $290000 \mathrm{~s}$. In particular interval II corresponds to the rather stable portion of the light curve, interval III corresponds to the central portion of the flare while the other two are the initial and final segments of the light curve. We had a large number of events in each of these intervals: the lowest number of MECS counts was about 35000 (interval IV), but we chose to exclude PDS data because of the low net signal in each interval. The resulting reduced $\chi^{2}$ values, given in Table 4 , are fully satisfactory and the spectral parameters are given in Table 5; the corresponding SEDs in the LECS and MECS energy bands are plotted in Fig. 8 with their best fits. Note that the curvature parameter $b$ changed very little from the mean value for the entire data set, whereas the normalisation $K$ changed by a factor of $\sim 2$. This result implies that the spectral changes were rather limited during the pointing, and in particular, the spectral curvature during the flare was the same measured in the other intervals. 


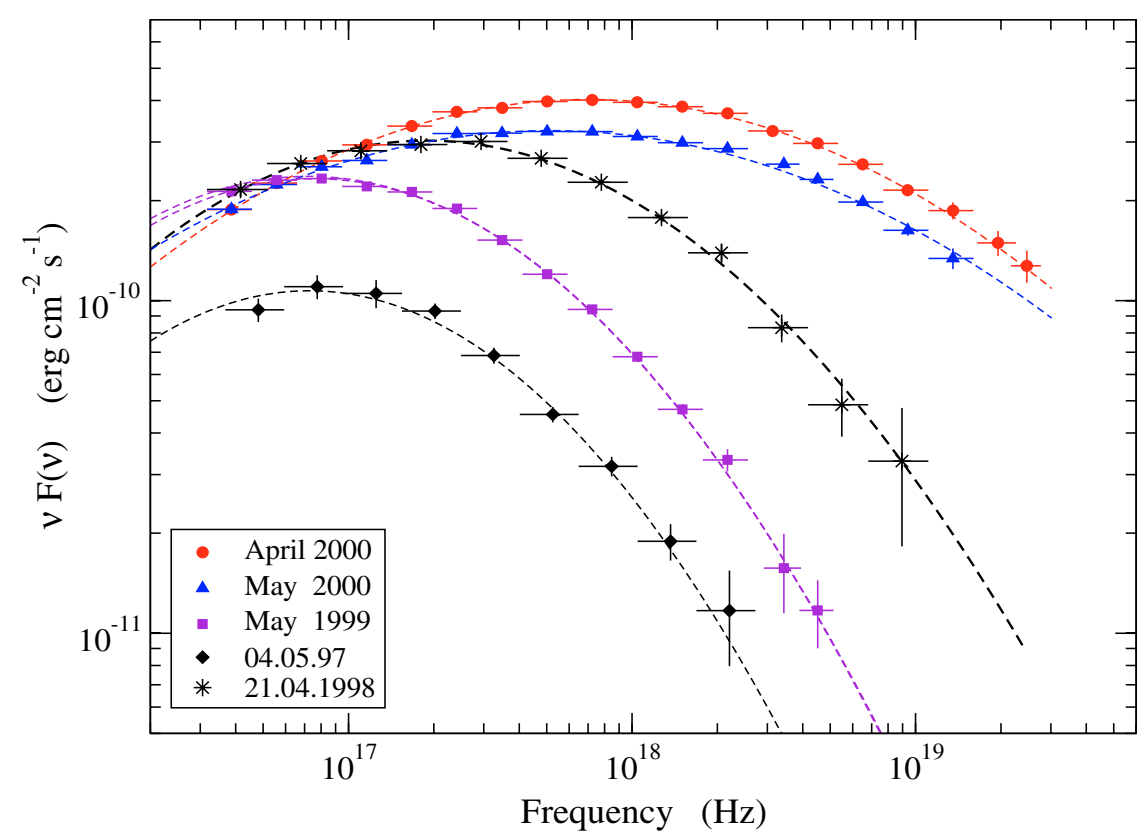

Fig. 7. The X-ray Spectral Energy Distributions of Mkn 421 in the long BeppoSAX observations of May 1999, April and May 2000. Best fit spectra (dashed lines) were obtained with a log-parabolic law. The SED of the two observations of 1997, May 4 and 1998, April 21 are also plotted to show the spectral evolution of the source.

Table 4. Reduced $\chi^{2}$ values for the best fit spectra of Mkn 421 with a power law with exponential cutoff and a log-parabola for the 1999-2000 observations.

\begin{tabular}{cccc}
\hline \hline Date & PL+EC & $\log P$ & d.o.f. \\
\hline 1999-05 & 4.16 & 1.11 & 141 \\
I & 1.57 & 1.11 & 111 \\
II & 3.22 & 1.14 & 111 \\
III & 2.51 & 1.06 & 111 \\
IV & 1.77 & 1.04 & 111 \\
& & & \\
& & & \\
2000-04 & 13.11 & 1.06 & 152 \\
H & 8.13 & 1.01 & 152 \\
F & 8.21 & 1.06 & 152 \\
& & & \\
2000-05 & 7.89 & 1.34 & 149 \\
I & 5.82 & 1.37 & 149 \\
II & 2.77 & 1.03 & 136 \\
III & 2.87 & 1.26 & 136 \\
IV & 2.14 & 0.87 & 136 \\
V & 1.67 & 1.00 & 121 \\
\hline
\end{tabular}

\subsection{The April 2000 observation}

In spring $2000 \mathrm{Mkn} 421$ reached the highest brightness level of all the BeppoSAX observations. The source was well detectable in the PDS at energies higher than $100 \mathrm{keV}$ and therefore we considered in our analysis the PDS $13-120 \mathrm{keV}$ data.

The resulting $\chi^{2}$ for the entire observation (see Table 4) was well acceptable and the corresponding log-parabolic SED in shown in Fig. 7. The source spectrum in this high state was very different from that observed in fainter states. In particular, the spectral curvature $b$ decreased to 0.21 , and the peak energy increased at about $3 \mathrm{keV}$.

From the light curve of Fig. 2 we see that the X-ray flux of Mkn 421 was characterised by an approximately oscillating behaviour with a typical amplitude of about 1.5 , without prominent flares. We selected then two data sets, useful for a more detailed analysis, on the base of the count rate. All the events in the time intervals during which the mean 2-10 keV MECS count rate was higher than $7.5 \mathrm{ct} / \mathrm{s}$ were included in the High state subset $\mathbf{H}$, while the remaining events were in the Faint subset $\mathbf{F}$. The parameters of the log-parabolic best fits of these two subsets are given in Table 5 and the corresponding SEDs are plotted in Fig. 9.

Again spectral changes between the two states are rather small: in particular the value of $b$ was practically unchanged while $a$ changed by 0.08 , implying an increase of the peak energy from 2.3 to $3.7 \mathrm{keV}$ in the $\mathbf{H}$ state.

\subsection{The May 2000 observation}

During the last observation of May 2000 Mkn 421 was in a high luminosity state. The spectral best fit for the entire data set was evaluated using the same energy ranges for LECS and MECS of April 2000 observation, while that of PDS was limited at $70 \mathrm{keV}$. The resulting reduced $\chi^{2}$ is reported in Table 4. The mean spectral continuum is very well described by the logparabolic law, as shown by the SED plotted in Fig. 7.

Also for this long pointing the spectral analysis was performed in five shorter segments, selected on the basis of the time features in the light curve of Fig. 3. Segment I, from the beginning to $180000 \mathrm{~s}$, includes the first portion of the light curve before the flare; segments II and III were taken in the rising and decaying portions of the flare; segment IV 
Table 5. Best fit spectral parameters of the log-parabolic model for the 1999 and 2000 observations of Mkn 421.

\begin{tabular}{|c|c|c|c|c|c|c|c|}
\hline Date & $a$ & $b$ & $K$ & $E_{\mathrm{p}}(\mathrm{keV})$ & $v_{\mathrm{p}} F\left(v_{\mathrm{p}}\right)^{(2)}$ & $F_{\text {bol }}^{(2)}$ & $F_{2-10 \mathrm{keV}}^{(2)}$ \\
\hline 1999-05-04/08 & $2.421(.004)$ & $0.419(.005)$ & $1.140(.007) \times 10^{-1}$ & $0.315(.006)$ & $2.33(.02)$ & $9.7(.1)$ & $1.10(.01)$ \\
\hline $1999-05 \mathrm{I}^{(1)}$ & $2.36(.01)$ & $0.39(.01)$ & $1.22 \quad(.02) \times 10^{-1}$ & $0.35 \quad(.01)$ & $2.37(.05)$ & $10.2(.2)$ & $1.31(.03)$ \\
\hline $1999-05 \mathrm{II}^{(1)}$ & $2.539(.006)$ & $0.442(.008)$ & $(.01) \times 10^{-1}$ & $0.25 \quad(.01)$ & $2.06(.03)$ & $8.3(.2)$ & $0.72(.02)$ \\
\hline $1999-05$ III $^{(1)}$ & $2.294(.007)$ & $0.450(.009)$ & $(.02) \times 10^{-1}$ & $0.47 \quad(.01)$ & $3.04(.04)$ & $12.2(.2)$ & $1.89(.03)$ \\
\hline 1999-05 IV (1) & $2.45 \quad(.01)$ & $0.45 \quad(.01)$ & $(.02) \times 10^{-1}$ & $0.32 \quad(.01)$ & $2.22(.05)$ & $8.9(.2)$ & $0.98(.02)$ \\
\hline $2000-04-26 / 30$ & $1.805(.002)$ & $0.212(.002)$ & $2.270(.007) \times 10^{-1}$ & $2.88(.04)$ & $4.03(.01)$ & $23.5(.1)$ & $6.25(.03)$ \\
\hline 2000-04 H & $1.765(.004)$ & $0.205(.003)$ & $2.59 \quad(.01) \times 10^{-1}$ & $3.7 \quad(.1)$ & $4.85(.03)$ & $28.8(.3)$ & $7.65(.08)$ \\
\hline $2000-04 \mathrm{~F}$ & $1.843(.003)$ & $0.222(.003)$ & $2.041(.008) \times 10^{-1}$ & $2.26(.04)$ & $3.49(.02)$ & $19.9(.2)$ & $5.27(.05)$ \\
\hline 2000-05-09/12 & $1.882(.003)$ & $0.180(.003)$ & $1.945(.006) \times 10^{-1}$ & $2.13(.05)$ & $3.26(.01)$ & $20.6(.2)$ & $4.95(.05)$ \\
\hline 2000-05 I & $1.962(.003)$ & $0.195(.003)$ & $1.734(.007) \times 10^{-1}$ & $1.25(.02)$ & $2.79(.01)$ & $17.0(.1)$ & $3.86(.02)$ \\
\hline 2000-05 II & $1.704(.009)$ & $0.200(.007)$ & $2.87 \quad(.03) \times 10^{-1}$ & $5.5 \quad(.4)$ & $5.9 \quad(.1)$ & $35.6(.9)$ & $9.32(.24)$ \\
\hline 2000-05 III & $1.737(.008)$ & $0.197(.007)$ & $(.02) \times 10^{-1}$ & $4.6 \quad(.3)$ & 5.1 & $31.1(.7)$ & $8.12(.18)$ \\
\hline 2000-05 IV & $1.72(.01)$ & $0.194(.009)$ & $(.03) \times 10^{-1}$ & $5.3 \quad(.5)$ & 5.7 & $34.7(.9)$ & $8.97(.23)$ \\
\hline $2000-05 \mathrm{~V}$ & $1.88 \quad(.01)$ & $0.19 \quad(.01)$ & $(.02) \times 10^{-1}$ & $2.1 \quad(.1)$ & $2.86(.04)$ & $17.7(.5)$ & $4.35(.13)$ \\
\hline
\end{tabular}

Errors are given at 1 sigma for one interesting parameter.

(1) PDS data not included.

(2) In units of $10^{-10} \mathrm{erg} \mathrm{cm}^{-2} \mathrm{~s}^{-1}$.

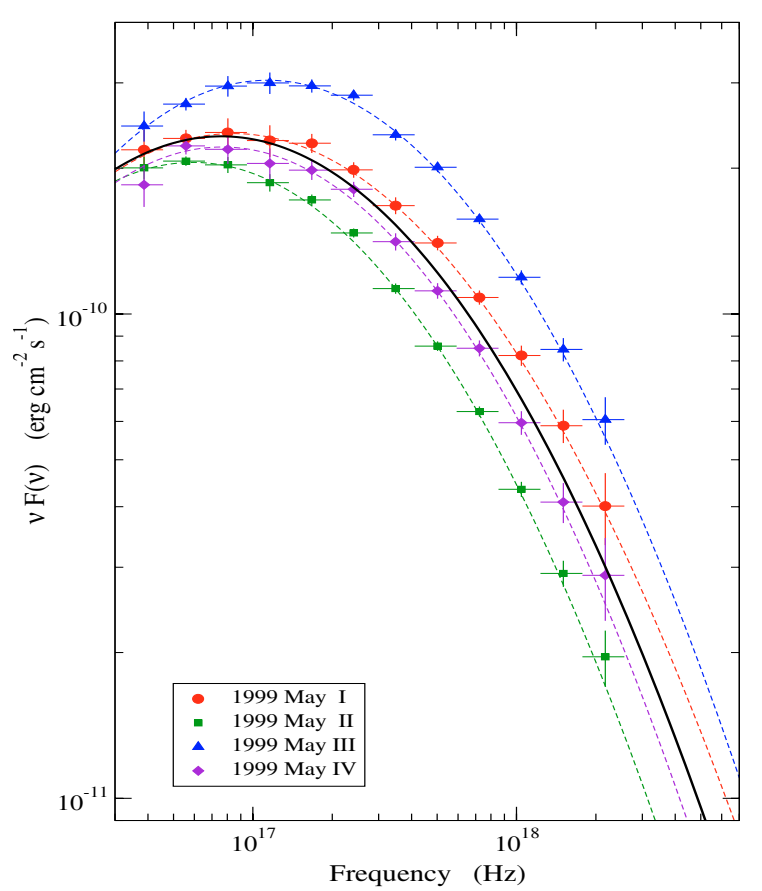

Fig. 8. The Spectral Energy Distributions of Mkn 421 in the four time segments of the May 1999 observation. Dashed lines are the logparabolic best fits, while the thick solid line is the best fit of the entire observation.

from 225000 to 250000 seconds contains the events of the second rapid flare; finally, the last segment $\mathrm{V}$ includes the remaining data to the end of the observation. Log-parabolic best fits, as expected, are generally good (see the reduced $\chi^{2}$ values in Table 4) and systematically much lower than those obtained with the PL+EC model. The resulting spectral parameters are

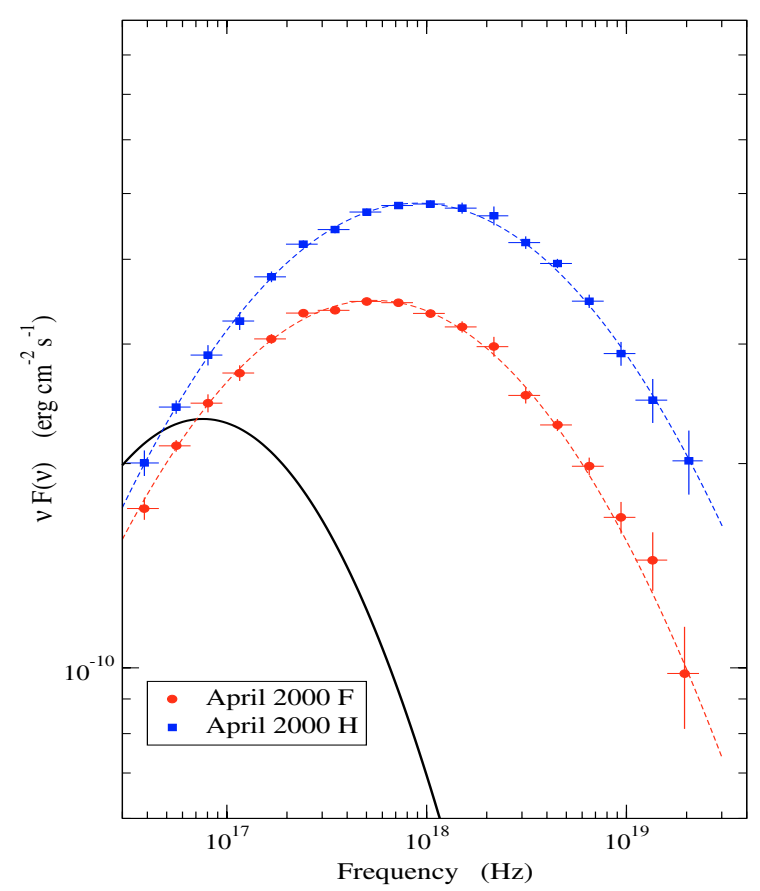

Fig. 9. The Spectral Energy Distributions of Mkn 421 in the High and Faint states during the April 2000 observation. Dashed lines are the log-parabolic best fits, the thick solid line is the best fit of the entire May 1999 observation, the same plotted in Fig. 8, shown for a better comparison among the different luminosity states of the source.

also given in Table 5, while the SEDs of the segments I, II, III are shown in Fig. 10. Those of the two remaining segments are very similar to them - in particular that of IV is intermediate between II and III and that of V is close to that of I - and have not been plotted to for simplifying the figure. We found that 


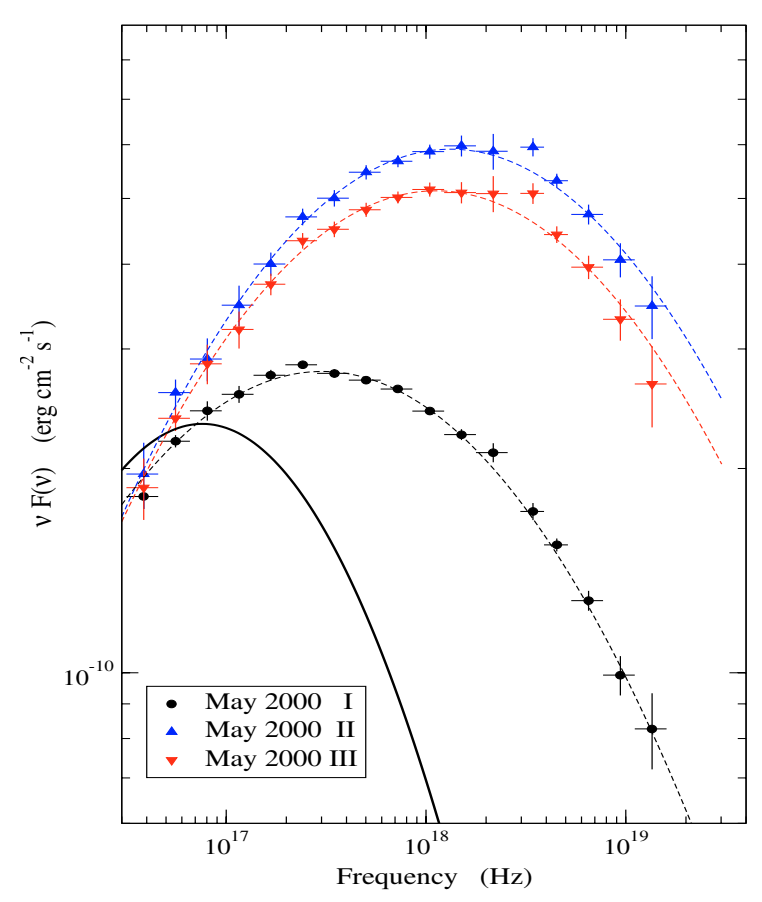

Fig. 10. The Spectral Energy Distributions of Mkn 421 in the first three time segments of the May 2000 observation. Dashed lines are the log-parabolic best fits. The thick solid line is the best fit of the entire May 1999 observation, the same plotted in Fig. 8, shown for a better comparison among the different luminosity states of the source.

the most stable parameter was $b$, remaining within the small range $0.19-0.20$, while for the entire observation $b$ was lower and equal to 0.18 . This apparent inconsistency is likely due to the superposition of different spectra with similar curvature but different peak energies (varying by a factor of $\sim 4$, see Fig. 10) which makes the overall spectrum appear less curved. This effect is indeed not apparent in the 1999 and April 2000 observations when the peak energies were more stable.

These results indicate that one of the most relevant change with respect to the 1997-1999 data is this large decrease of the spectral curvature, likely associated with the much higher luminosity of the source. However, note that $b$ did not change greatly on the time scale of weeks from April to May 2000.

\section{Statistical particle acceleration and log-parabolic spectra}

In the previous sections we have shown that the log-parabolic law is useful to model the rather mild spectral curvature observed in the X-ray emission of Mkn 421 and in other BL Lac sources. It is important to understand if this law is a simple mathematical tool to describe curved shapes or if it can be explained by means of some physical process. In this section we present some phenomenological considerations on the particle spectra from statistical acceleration. We will show that it is possible to obtain an integral energy distribution for the particles that follows a log-parabolic law under quite reasonable hypotheses and derive some simple relations between the spectral parameters and the most relevant factors affecting the acceleration. In the Sect. 6.1 we will give only a general trace and not a theory of energy dependent acceleration. More detailed analytical and numerical calculations are necessary to confirm the main findings of this approach, and these mathematical developments will be the subject of forthcoming works.

\subsection{Energy distribution of accelerated particles}

The energy spectrum of accelerated particles by some statistical mechanism, like that occurring in a shock wave or in a strong perturbation moving down a jet, is usually written as a power law:

$N(>\gamma)=N_{0}\left(\gamma / \gamma_{0}\right)^{-s+1}$,

where $N(>\gamma)$ is the number of particles having a Lorentz factor greater than $\gamma$ and $s$ is the spectral index given by:

$s=-\frac{\log p}{\log \varepsilon}+1$,

here $p$ is the probability that a particle undergoes an acceleration step $i$ in which it has an energy gain equal to $\varepsilon$, generally assumed both independent of energy:

$\gamma_{i}=\varepsilon \gamma_{i-1}$

and

$N_{i}=p N_{i-1}=N_{0} p^{i}$.

A log-parabolic energy spectrum follows when the condition that $p$ is independent of energy is released and one assumes that it can be described by a power relation as:

$p_{i}=g / \gamma_{i}^{q}$

where $g$ and $q$ are positive constants; in particular, for $q>0$ the probability for a particle to be accelerated is lower when its energy increases. Such a situation can occur, for instance, when particles are confined by a magnetic field with a confinement efficiency decreasing for an increasing gyration radius. After simple calculations one finds:

$N_{i}=N_{0} \frac{g^{i}}{\prod_{j=0}^{i-1} \gamma_{j}^{q}}$.

Using Eq. (12) one can write the product on the right side as:

$\prod_{j=0}^{i-1} \gamma_{j}^{q}=\gamma_{0}^{i q} \prod_{j=1}^{i-1} \varepsilon^{j q}=\gamma_{0}^{i q}\left(\varepsilon^{q}\right)^{i(i-1) / 2}$

where $\gamma_{0}$ is the initial Lorentz factor of the particles; inserting this result into Eq. (15) we obtain:

$N_{i}=N_{0}\left(\frac{g}{\gamma_{0}^{q}}\right)^{i}\left(\varepsilon^{q}\right)^{-i(i-1) / 2}$.

Finally, combining this equation with Eq. (12) one can obtain the integral energy distribution of the accelerated particles, which is a log-parabolic law:

$N(>\gamma)=N_{0}\left(\gamma / \gamma_{0}\right)^{-\left[s-1+r \log \left(\gamma / \gamma_{0}\right)\right]}$, 
with

$s=-\frac{\log \left(g / \gamma_{0}\right)}{\log \varepsilon}-\frac{q-2}{2}$

and

$r=\frac{q}{2 \log \varepsilon}$.

The differential spectrum $N(\gamma)$ is:

$$
\begin{aligned}
N(\gamma) & =\frac{N}{\gamma} \frac{\mathrm{d} \log N}{\mathrm{~d} \log \left(\gamma / \gamma_{0}\right)} \\
& =\frac{N_{0}}{\gamma_{0}}\left|s-1+2 r \log \left(\gamma / \gamma_{0}\right)\right|\left(\gamma / \gamma_{0}\right)^{-s-r \log \left(\gamma / \gamma_{0}\right)} .
\end{aligned}
$$

This is not a log-parabolic law, but differs from it very little, because of a factor only logarithmic dependent on the particle energy. Numerical calculations show that the differences between this law and a log-parabolic one is smaller than $10 \%$ over a several decade wide energy range. Practically, the spectral curvature corresponding to Eq. (21) is very similar to that of a log-parabola and cannot be distinguished in a spectral analysis like that we presented in previous sections. In the following we will assume, therefore, that the differential energy distribution of accelerated particles is well approximated by a log-parabola like that results from approximating the log term as a constant which can be included in the normalisation.

It is important to note that the spectral parameters given by Eqs. (19) and (20) are in a linear relationship; in fact, after eliminating $\log \varepsilon$ one obtains:

$s=-r\left(\frac{2}{q} \log \left(g / \gamma_{0}\right)\right)-\frac{q-2}{2}$.

The assumption of Eq. (14) about the energy dependence of the acceleration probability can been modified to take into account other escape processes. For instance, one can assume that the acceleration probability is constant for low energies and begins to decrease above a critical Lorentz factor $\gamma_{\mathrm{c}}$; a phenomenological formula for this can be:

$p_{i}=\frac{g}{1+\left(\gamma_{i} / \gamma_{\mathrm{c}}\right)^{q}}$.

The asymptotic behaviour of the particle energy distribution derived from such an assumption can easily be devised: for $\gamma \ll \gamma_{\mathrm{c}}$ it will follow a power law with spectral in$\operatorname{dex} s=-(\log g) /(\log \varepsilon)$, while for $\gamma \gg \gamma_{\mathrm{c}}$ it will approximate a log-parabolic spectrum like Eq. (18). We expect that in this case the spectrum of emitted radiation can be described by Eq. (9).

\subsection{Synchrotron radiation spectrum}

It is important to know the relations between the spectral parameters $a$ and $b$ of the emitted radiation and those of the electron population, namely $s$ and $r$. The spectral distribution of the SR emitted by relativistic electrons with a log-parabolic energy distribution cannot be computed analytically in a simple way, however, for our aim the relations between the spectral indices can be derived under the usual $\delta$-approximation and the assumption that the electrons are isotropically distributed in a homogeneous randomly oriented magnetic field:

$P_{S}(v)=\int P(v(\gamma)) N(\gamma) \mathrm{d} \gamma$

where the power radiated by a single particle is:

$P(v)=C_{1} \gamma^{2} B^{2} \delta\left(v-v_{S}\right)$

and

$v_{S}=C_{2} \gamma^{2} B=\gamma^{2} v_{0}$

where $C_{1}=\left(4 e^{4}\right) /\left(9 m^{2} c^{3}\right), C_{2}=0.29(3 e) /(4 \pi m c)$ (Rybicki \& Lightman 1979).

From Eq. (20) then follows:

$P_{S}(v) \propto\left(v / v_{0}\right)^{-\left(a+b \log \left(v / v_{0}\right)\right)}$

with

$a=(s-1) / 2$

$b=r / 4$.

It is important to stress that the parameter $a$, as defined above, is different from that we defined in Eq. (3): the former being the energy index at the frequency $v_{0}$, whereas the latter the photon index at the energy $E_{1}$, that in our analysis has been fixed at $1 \mathrm{keV}$, not corresponding to the frequency $v_{0}$. It is not difficult to verify that these two parameters differ for an additional constant, whose value depends on $v_{0}$ and $v_{1}=E_{1} / h$. We stress, however, that Eq. (22) implies that also $a$ and $b$ are expected to be linearly dependent on each other.

\section{Discussion}

The interpretation of the spectral variability of BL Lac objects is not a simple problem because it involves the description of non stable processes with many unknown physical quantities. It is important, therefore, in the spectral data analysis, to use models characterised by rather simple analytical formulae which can be directly related to some physical parameters of the source. In our analysis of the BeppoSAX wide band observations of Mkn 421, covering in some occasions about three decades in energy, we used a log-parabolic spectral model which represents a further step in this direction. We have shown that the curved spectra of these sources are very well represented by a law of this type, as opposed to power laws with exponential cut-off for which we did not obtain generally acceptable fits.

As mentioned in the Introduction the fact that the mild curvature of blazar spectra can be well described by a logparabolic law was noticed for the first time by Landau et al. (1986), but these authors did not give a satisfactory model justification for it. A widely accepted interpretation of the spectral curvature is in terms of radiation cooling of high energy electron population, injected with a power law spectrum, via synchrotron and inverse Compton processes. In rapidly variable sources, like blazars, there are several processes and time scales which compete in determining the observed light curves and 
spectral shapes (see, for instance, Massaro et al. 1996): high energy particle acceleration, injection and diffusion through the emitting volume, radiative cooling, radiation crossing time and particle escape are all contributing processes which may be associated with different time scales and it is not simple to establish which is the dominant one that determines the observed variability. In the case of HBL sources, in which the synchrotron emission peaks in the $\mathrm{X}$ rays the radiative cooling time can be very short:

$$
\begin{aligned}
t_{\mathrm{rad}} & =3.39 \times 10^{10}\left(B^{1 / 2} / u\right) v^{-1 / 2} \\
& <8.52 \times 10^{11} B^{-3 / 2} v^{-1 / 2} \mathrm{~s},
\end{aligned}
$$

where $u=\left(B^{2} / 8 \pi\right)+w_{\mathrm{ph}}$ is the energy density of the magnetic and photon fields ( $B$ in Gauss), the upper limit in Eq. (29) corresponds to neglecting the photon energy density.

In the bulk frame of the electrons moving down the jet, for frequencies $v \simeq 10^{16} \mathrm{~Hz}$ (we assume a beaming factor of about 10) and an effective magnetic field of $\sim 1$ Gauss, we obtain radiative lifetimes of the order of one hour, corresponding to minutes in the observer's frame. The emission can be maintained only if the acceleration mechanisms are continuously at work.

The most common approach to produce curved spectra is to consider radiative losses and the escape of high energy electrons from the emitting region: the literature has been summarized, for the other TeV blazar Mkn 501, in the recent paper by Krawczynski et al. (2002). The intrinsic difficulty is that numerical calculations are necessary to solve the transfer equation of the electrons and consequently it is hard to introduce a parameter for describing the spectral curvature and to find how it is related to the other main physical parameters of the model.

We followed a quite different approach, based on the hypothesis that the particle spectrum is curved from injection. In Sect. 6 we have shown that log-parabolic spectra are naturally obtained for an energy dependent probability of statistical acceleration and derived how the curvature parameter $b$ can be simply related to the acceleration gain $\varepsilon$. The fact that the spectra of the synchrotron component of several BL Lac objects have a log-parabolic shape with a rather narrow range of $b$ values (see, for instance the SED catalog by Giommi et al. 2002) strongly suggests that it can be a "general" characteristic of these sources. It is unlikely that acceleration, loss and escape processes combine to produce such similar spectra.

In a simplified dimensional approach the transfer equation can be written as:

$\frac{N}{t}+\frac{N}{t_{\mathrm{rad}}(\gamma)}=Q(\gamma)$

in which only the radiative cooling has been considered and where $Q(\gamma)$ is the source function. In the limit of a very long cooling we obtain $N(\gamma, t) \simeq Q(\gamma) t$, while for a very fast cooling one has $N(\gamma, t) \simeq Q(\gamma) t_{\text {rad }} \propto Q(\gamma) / \gamma$. We see, therefore, that in the case of an injected spectrum given by a log-parabolic law, the equilibrium particle spectrum has the same curvature parameter while the constant index $s$ is increased by unity.

An interesting check if the log-parabolic curve is actually related to the statistical acceleration is the existence of a linear relation between the two spectral parameters $a$ and $b$, as a

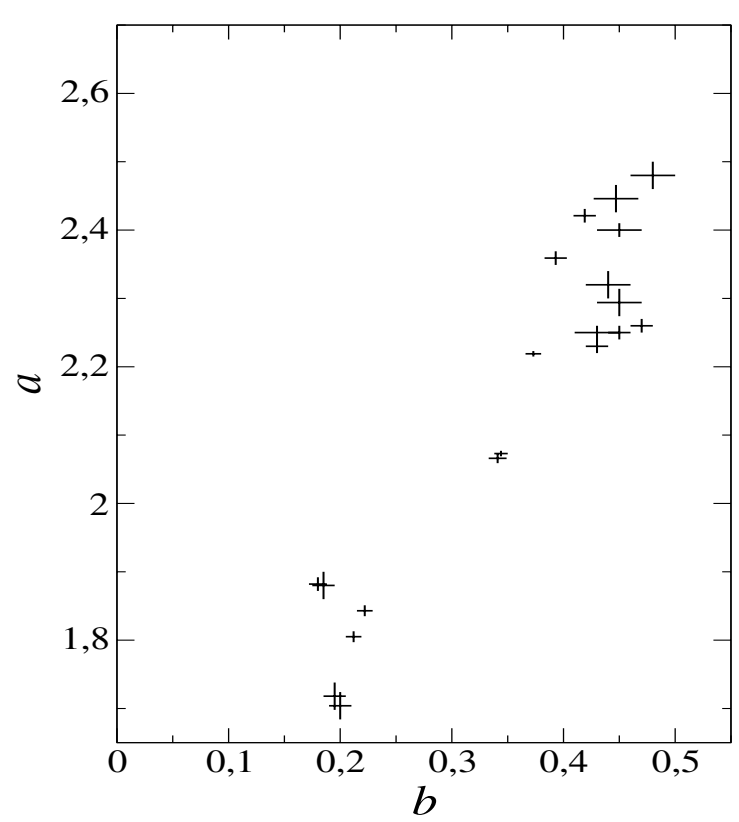

Fig. 11. The high correlation between the two spectral parameters $a$ and $b$ of the log-parabolic law supports the energy dependent statistical acceleration of the emitting electrons.

consequence of that given by Eq. (22). In Fig. 11 we plotted the points corresponding to the best fit values given in Tables 3 and 5. It is evident that the two quantities are positively correlated with a linear correlation coefficient equal to 0.940 . This result can be considered a further relevant indication supporting the hypothesis that the curvature is caused by the particle acceleration process. High luminosity states are then related to a greater acceleration gain $\varepsilon$. In our analysis we measured a change of $b$ from $\sim 0.45$ to $\sim 0.20$, their ratio would then be equal to that of the logarithms of the acceleration gains in the two states - see Eqs. (20) and (27). We can therefore estimate that in the high state the acceleration gain was

$\varepsilon_{\mathrm{h}}=\varepsilon_{\mathrm{f}}^{\left(b_{\mathrm{f}} / b_{\mathrm{h}}\right)}$,

where " $\mathrm{f}$ " and " $\mathrm{h}$ " indicate the faint and high state, respectively. With the above $b$ values, we have $\varepsilon_{\mathrm{h}}=\varepsilon_{\mathrm{f}}^{2.2}$, thus for $\varepsilon_{\mathrm{f}} \sim$ $1.2-1.5$, we obtain that in the high state the acceleration efficiency would increase by about $50 \%$, and does not require relevant modifications of the source structure.

Another important consequence of the log-parabolic modelling of blazar's SEDs is that it can be useful to distinguish the possible presence of several emission components at the same time. The optical-X-ray SED of Mkn 421 in the April 1998 observation (Fig. 5) indicates that simple extrapolations of the $\mathrm{X}$-ray spectra down to the optical range does not match nearly simultaneous data. Furthermore, the light curves plotted in Figs. 1-3 show that the amplitude of the variations is much higher at energies higher than $2 \mathrm{keV}$ than below. This behaviour is naturally explained if the emission in the soft $\mathrm{X}$ rays is due to the superposition of two components: one associated with the slowly variable optical-UV emission and the other, dominating above $\sim 1 \mathrm{keV}$, responsible for the larger brightness changes.

Disentangling different emission components, on the basis of SED structure and variability, can be achieved only with 
coordinated observational campaigns covering a very wide spectral range, from IR to high energy $\gamma$ rays. This is certainly one of the most important scientific targets for future high energy astrophysics space observatories (e.g. Swift, AGILE and GLAST) and it is important to organize this work in advance to obtain very fruitful results.

Acknowledgements. This work is based on BeppoSAX data available from the public archive at the ASI Science Data Center. The authors are grateful to F. Fiore for useful suggestion on the data analysis and to A. Cavaliere and G. Ghisellini for fruitful discussions on the modelling of BL Lac objects. The CNR Institutes and the BeppoSAX Science Data Center are financially supported by the Italian Space Agency (ASI) in the framework of the BeppoSAX mission. Part of this work was performed with the financial support Italian MIUR (Ministero dell' Istruzione Universitá e Ricerca) under the grant Cofin 2001/028773.

\section{References}

Boella, G., Chiappetti, L., Conti, G., et al. 1997b, A\&AS, 122, 327 Fiore, F., Guainazzi, M., \& Grandi, P. 1999, Cookbook for BeppoSAX NFI Spectral Analysis (http://www.sdc.asi.it/software)

Fossati, G., Celotti, A., Chiaberge, M., et al. 2000a, ApJ, 541, 153

Fossati, G., Celotti, A., Chiaberge, M., et al. 2000b, ApJ, 541, 166

Fukugita, M., Shimasaku, K., \& Ichikawa, T. 1995, PASP, 107, 945

Giommi, P., Capalbi, M., Fiocchi, M., et al. 2002, Proc. Blazar Astrophysics with BeppoSAX and Other Observatories, ed. P. Giommi, E. Massaro, \& G. G. C. Palumbo, ASI Special Publication, 63
Inoue, S., \& Takahara, F. 1996, ApJ, 97, 1

Krawczynski, H., Coppi, P. S., \& Aharonian, F. 2002, MNRAS, 336, 721

Krennrich, F., Biller, S. D., Bind, I. H., et al. 1999, ApJ, 511, 149

Landau, R., Golisch, B., Jones, T., et al. 1986, ApJ, 308, 78

Lockman, F. J., \& Savage, B. D. 1995, ApJS, 97, 1

Malizia A., Capalbi, M., Fiore, F., et al. 2000, MNRAS, 312, 123

Massaro, E., Nesci, R., Maesano, M., et al. 1996, A\&A, 314, 87

Massaro, E., Cusumano, G., Litterio, M., \& Mineo, T. 2000, A\&A, 361,695

Massaro, E., Giommi, P., Perri, M., et al. 2003, A\&A, 399, 33

Mead, A. R. G., Ballard, K. R., Brand, P. W. J. L., et al. 1990, A\&AS, 83,183

Orr, A., Parmar, A., Guainazzi, M., Fiore, F., \& Ricci, D. 1997, BeppoSAX Science Data Center Technical Report No 15

Padovani, P., \& Giommi, P. 1995, ApJ, 111, 222

Parmar, A. N., Martin, D. D. E., Bavdaz, M., et al. 1997, A\&AS, 122, 309

Perri, M., Massaro, E., Giommi, P., et al. 2003, A\&A, 407, 453

Pian, E., Vacanti, G., Tagliaferri, G., et al. 1998, ApJ, 492, L17

Punch, M., Akerlof, C. W., Cawley, M. F., et al. 1992, Nature, 358, 477

Rybicki, G. B., \& Lightman, A. P. 1979, Radiative Processes in Astrophysics (New York: Wiley)

Tanihata, C., Takahashi, T., Kataoka, J., \& Madejski, G. M. 2003, ApJ, 584,153

Tosti, G., Fiorucci, M., Luciani, M., et al. 1998, A\&A, 339, 41

Urry, C. M., Scarpa, R., O’Dowd, M., et al. 2000, ApJ, 532, 816

Villata, M., Raiteri, C. M., Lanteri, L., et al. 1998, A\&AS, 130, 305

Zhang, Y. H. 2002, MNRAS, 337, 609 